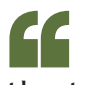

the tested

proteins

reduced oocyst

numbers in

two different

mosquito

species

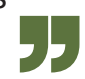

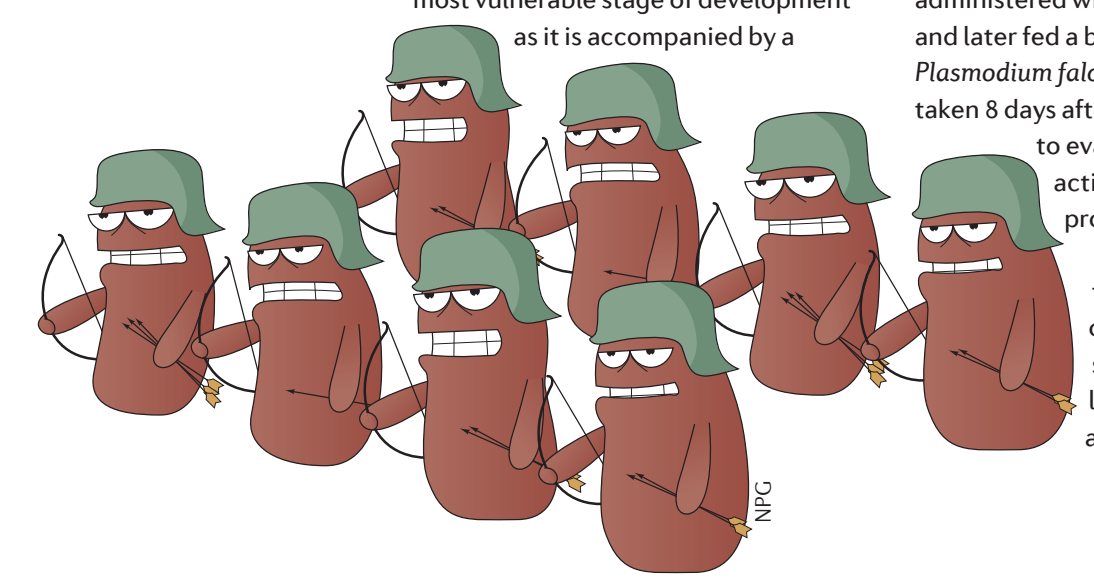
as it is accompanied by a

\title{
ANTIMICROBIALS
}

\section{Arming symbionts with antimalarials}

As the frequencies of mosquito insecticide resistance and parasite drug resistance continue to rise, alternative strategies to curb the emergence and spread of malaria are urgently needed. Previous efforts have focused on genetically modifying the mosquito vector to resist infection with Plasmodium spp.; however, this has been difficult to achieve owing to technical challenges. Now, Wang et al. report the engineering of a mosquito symbiont to secrete anti-Plasmodium effector proteins and show that this strategy effectively impedes parasite development in the insect.

During their life cycle,

Plasmodium spp. transition through various developmental stages in vector mosquitoes, where differentiation from ookinetes to oocysts occurs in the midgut. This is considered to be the most vulnerable stage of development population bottleneck, which sees a severe drop in the number of parasites. The bacterium Pantoea agglomerans is a common member of the mosquito midgut microbiota, and its numbers rapidly expand after ingestion of a blood meal. Thus, by introducing transgenes encoding anti-Plasmodium effectors into P. agglomerans, the authors aimed to maximize exposure of the parasite to these inhibitory proteins at a time and place at which it is most sensitive to killing.

Plasmids encoding one of a range of effector proteins were individually transformed into P. agglomerans, along with a second plasmid encoding the Escherichia coli haemolysin A system, which facilitated secretion of the effectors from the engineered bacteria. To examine the effectiveness of this strategy in vivo, mosquitoes were administered with recombinant bacteria and later fed a blood meal containing Plasmodium falciparum. Oocyst counts ys after infection were used o evaluate the inhibitory action of each effector protein.

Although all of the tested effectors reduced oocyst numbers, a scorpion antimalaria lytic peptide (scorpine) and a fusion peptide composed of a chitinase propeptide and four copies of the Plasmodium enolase-plasminogen interaction peptide demonstrated the most potent inhibition and reduced oocyst formation by $\sim 98 \%$. Furthermore, the infection prevalence (the proportion of mosquitoes carrying oocysts) was reduced by up to $84 \%$ when scorpine-secreting bacteria were present. Finally, the authors found that each of the tested proteins reduced oocyst numbers in two different mosquito species and that they were also active against the rodent parasite Plasmodium berghei, indicating that this strategy may be a universal solution for the control of malaria.

These data demonstrate how the microbiota can be manipulated to provide a powerful weapon against pathogens. However, technical obstacles, such as devising an efficient strategy for the colonization of wild mosquitoes with engineered symbionts, as well as controversies surrounding the release of genetically modified organisms into the environment, remain to be addressed.

Christina Tobin Kåhrström

ORIGINAL RESEARCH PAPER Wang, S. et al. Fighting malaria with engineered symbiotic bacteria from vector mosquitoes. Proc. Natl Acad. Sci. USA 16 Jul 2012 (doi:10.1073/pnas.1204158109) 